\title{
Antibiotic treatment partially protects against type 1 diabetes in the Bio-Breeding diabetes-prone rat. Is the gut flora involved in the development of type 1 diabetes?
}

\author{
S. Brugman • F. A. Klatter • J. T. J. Visser • \\ A. C. M. Wildeboer-Veloo • H. J. M. Harmsen • \\ J. Rozing $\cdot$ N. A. Bos
}

Received: 17 February 2006 / Accepted: 9 May 2006 / Published online: 1 July 2006

(C) Springer-Verlag 2006

\begin{abstract}
Aims/hypothesis Accumulating data suggest that the gut immune system plays a role in the development of type 1 diabetes. The intestinal flora is essential for the development of the (gut) immune system and the establishment of tolerance. It has been reported that oral administration of food and bacterial antigens early in life suppresses later development of diabetes in the Bio-Breeding diabetesprone (BB-DP) rat. This study was designed to investigate the possible relationship between the development of diabetes and the composition of intestinal flora.

Materials and methods The intestinal flora of BB-DP rats, a rat model for type 1 diabetes, was characterised long before the clinical onset of diabetes by fluorescent in situ hybridisation. In a separate experiment, BB-DP rats were treated with antibiotics and the effect on diabetes incidence and level of insulitis was analysed.

Results We observed a difference in bacterial composition between rats that eventually did and those that did not develop diabetes. This difference was detectable long before clinical onset of the disease. Rats that did not develop diabetes at a later age displayed a lower amount of Bacteroides sp. Modulation of the intestinal flora through
\end{abstract}

\footnotetext{
S. Brugman · F. A. Klatter · J. T. J. Visser · J. Rozing •

N. A. Bos $(\triangle)$

Department of Cell Biology, Immunology Section,

University Medical Center Groningen,

University of Groningen,

A. Deusinglaan 1 ,

9713 AV Groningen, The Netherlands

e-mail: n.a.bos@med.umcg.nl
}

\footnotetext{
A. C. M. Wildeboer-Veloo · H. J. M. Harmsen

Department of Medical Microbiology,

University Medical Center Groningen,

Groningen, The Netherlands
}

antibiotic treatment decreased the incidence and delayed the onset of diabetes. A combination of antibiotic treatment and a protective hydrolysed casein diet completely prevented diabetes in the BB-DP rat.

Conclusions/interpretation Our data suggest that the intestinal flora is involved in the development of type 1 diabetes. Factors influencing composition of the intestinal flora could be a target for therapeutic intervention.

Keywords Antibiotics · BB-DP rats · Insulitis · Intestinal flora Type 1 diabetes
Abbreviations
BB-DP Bio-Breeding diabetes-prone
CON Conventional plant-based
FISH Fluorescent in situ hybridisation
HC Hydrolysed casein

\section{Introduction}

Type 1 diabetes results from complex interactions between genetic and environmentally determined factors. Experiments performed in animal models for type 1 diabetes have shown that diet can influence the diabetes outcome. Plantbased diets appear to be the most 'diabetogenic' and this seems to be dependent on dose ('antigenic load'). Hydrolysed casein (HC) diet, a diet in which the sole protein source is hydrolysed casein, has a protective effect on diabetes development in Bio-Breeding diabetes-prone (BBDP) rats [1]. Several mechanisms for the protective effects of the HC diet have been described, such as Th2 skewing, islet neogenesis and inhibition of hyperexpression of MHC class I on beta cells [2]. In addition, a diabetes-preventive 
gluten-free diet has been shown to decrease the number of caecal bacteria in non-obese diabetic mice, suggesting that such diets may also exert their protective effect by changing the intestinal flora $[3,4]$.

The fact that some BB-DP rats do not develop diabetes suggests that other factors, besides genes and diet, play a role in the development of diabetes. The increasing prevalence of food allergies in Western societies has led to the hypothesis that increased hygienic conditions throughout life can disturb normal tolerance induction to otherwise harmless food proteins and other (inhaled) antigens. Previous studies have shown that modulation of antigen exposure early in life can change the diabetes incidence in BB-DP rats [5, 6]. Furthermore, the antistaphylococcal drug fusidin was able to decrease the diabetes incidence in BB-DP rats [7].

In order to determine if the intestinal flora plays a role in the development of type 1 diabetes in the BB-DP rat, we analysed the intestinal flora of BB-DP rats that became diabetic and of BB-DP rats that did not become diabetic. This analysis was done long before the onset of diabetes in affected animals. In a separate experiment, we also modulated the intestinal flora by antibiotic treatment and determined the effect on the diabetes incidence and level of insulitis.

\section{Materials and methods}

Animals

BB-DP rats were derived from the Worchester BB-DP strain, but were bred in the Groningen animal facility (BBDPWor/ Gro). In our colony, 80 to $90 \%$ of the BB-DP rats spontaneously develop diabetes from 65 days of age on a conventional plant-based (CON) diet. No sex-related differences are observed. An animal is considered diabetic when blood glucose levels exceed $15 \mathrm{mmol} / \mathrm{l}$. Animals were treated in compliance with Dutch law on experimental animal care.

Experiment 1. Faecal flora composition of BB-DP rats before onset of diabetes

BB-DP rats, males and females, were group-housed (two or three/cage) under specific-pathogen-free conditions. Rats had free access to a CON diet (Rmh-B2181; Hope Farms, Woerden, the Netherlands) and water.

\section{Fluorescent in situ hybridisation (FISH)}

Faecal samples of 40-day-old BB-DP rats were dissolved in PBS, centrifuged at low speed and supernatants were fixed in $4 \%$ paraformaldehyde overnight at $4^{\circ} \mathrm{C}$. Samples were washed and fixed in $96 \%$ ethanol for $2 \mathrm{~h}$ at $4^{\circ} \mathrm{C}$.
Subsequently, samples were hybridised at $50^{\circ} \mathrm{C}$ with fluorescent (FITC)-labelled 16S rRNA-targeted oligonucleotide probes for different groups of bacteria (Eurogentec, Maastricht, the Netherlands) in hybridisation buffer (20 mmol/1 Tris- $\mathrm{HCl}, 0.9 \mathrm{~mol} / 1 \mathrm{NaCl}, 0.1 \%$ SDS, pH 7.2) overnight. The probes used were: Nonbact338 (negative control), Bact338 (universal), Bac303 (Bacteroides sp.), Erec482 (Clostridium sp.), Mib661 (mouse intestinal bacteria) and Lab158 (Lactobacillus sp.) [8,9]. Prior to analysis, samples were washed with hybridisation buffer without SDS for $30 \mathrm{~min}$ at $50^{\circ} \mathrm{C}$. Next, samples were washed with PBS, stained with propidium iodide (DNA stain) and analysed by flow cytometry [8].

Experiment 2. Antibiotic treatment of BB-DP rats

BB-DP rats, males and females, were group-housed (two or three/cage) under specific-pathogen-free conditions. Rats had free access to food and water (with or without antibiotics). BB-DP rats that received antibiotic treatment were kept in a laminar flow in filter-top cages.

\section{Treatment groups}

From weaning (day 21), two groups of BB-DP rats continuously received antibiotics in the drinking water (Bactrimel [sulphamethoxazole $(1.2 \mathrm{~g} / \mathrm{l})$ and trimethoprim $(240 \mathrm{mg} / \mathrm{l})$ ]; Roche, Woerden, the Netherlands) and colistine sulphate $(1.0 \mathrm{~g} / \mathrm{l})$, decreasing various Gram-negative and Gram-positive species. One group of rats on antibiotic treatment received a CON diet $(n=8)$, the other group received an HC diet $(n=10)$ (a modification of the AIN-93G diet containing 20\% hydrolysed casein as the source of amino acids [Solka-Floc; Teklad, Madison, WI, USA]). Two diet control groups that did not receive antibiotics were added (CON $n=14$; HC $n=15$ ).

\section{FISH and fluorescent microscopy}

In order to evaluate the effect of the antibiotic treatment on the amount of different bacteria, FISH was performed with the same set of probes as in Experiment 1. The bacteria hybridising with the probes were quantified by fluorescent microscopy.

Insulitis

Haematoxylin/eosin-stained paraffin sections of the pancreas were evaluated for insulitis as previously described [4].

Statistics

Student's $t$-test was used for statistical analysis of the intestinal flora composition and statistical analysis of the 
Fig. 1 Composition of the faecal flora of BB-DP rats (40 days old) expressed as a percentage of Bact338 binding. Black bars BB-DP rats that eventually developed diabetes $(n=22)$. White bars BB-DP rats that did not develop diabetes $(n=13)$. ${ }^{*} p=0.01$, Student's $t$-test, twotailed. Error bars, SEM

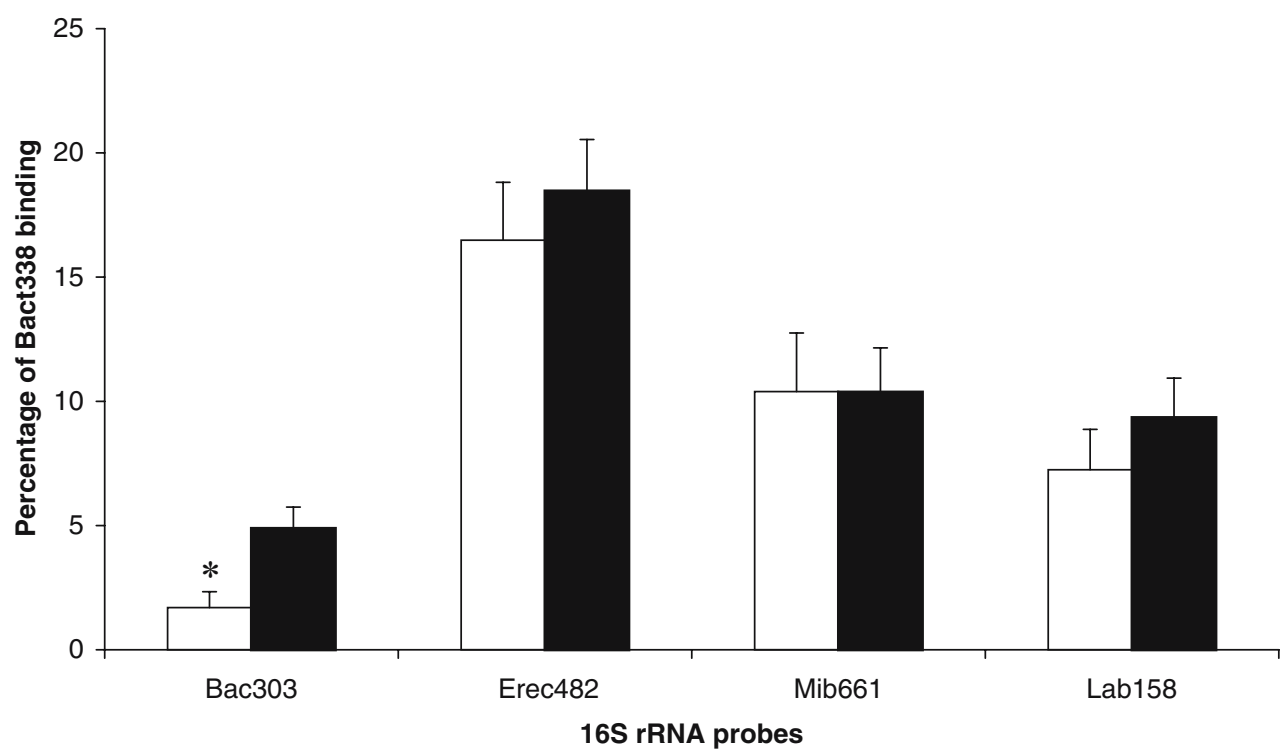

day of diabetes onset. The Kaplan-Meier test for survival, log-rank, was used for analysis of the survival data.

\section{Results}

Experiment 1. Faecal flora composition of BB-DP rats before onset of diabetes

In a retrospective analysis, we investigated whether differences in faecal flora composition already existed, prior to the disease onset, between BB-DP rats that eventually developed diabetes and rats that did not develop diabetes. Figure 1 shows binding of the bacterial group-specific probes in faecal samples at the pre-diabetic timepoint (day
40 of age). The faecal samples, taken before clinical onset, of rats that later developed diabetes show a higher binding to the Bacteroides sp. group-specific probe than the rats that did not develop diabetes $(p=0.01)$.

Experiment 2. Antibiotic treatment of BB-DP rats

\section{Effectiveness of the antibiotic treatment}

Antibiotic treatment lowered the amount of intestinal bacteria from $10^{10} / \mathrm{g}$ faeces to $10^{8} / \mathrm{g}$ faeces as measured by counting Bact338 (universal $16 \mathrm{~S}$ rRNA probe) positive events by fluorescent microscopy. Antibiotic treatment reduced binding to the specific probes. A reduction of 43 ,
Fig. 2 Diabetes incidence of different treatment groups. Solid line $\mathrm{BB}-\mathrm{DP}$ rats receiving $\mathrm{CON}$ $(n=14)$; dotted lineBB-DP rats receiving $\mathrm{HC}$ diet $(n=15)$; dashed line BB-DP rats on antibiotic treatment from weaning and receiving $\mathrm{CON}(n=8)$; dotted and dashed line BB-DP rats on antibiotic treatment from weaning and receiving $\mathrm{HC}$ diet $(n=10) .{ }^{*} p=0.01$ for the HC diet +antibiotic treatment compared with the $\mathrm{HC}$ diet alone; $* * p=0.002$ for the $\mathrm{HC}$ diet +antibiotic treatment compared with $\mathrm{CON}$ alone; ${ }^{* * *} p=0.02$ for the $\mathrm{CON}+$ antibiotic treatment compared with CON alone (all by Kaplan-Meier test for survival, log-rank)

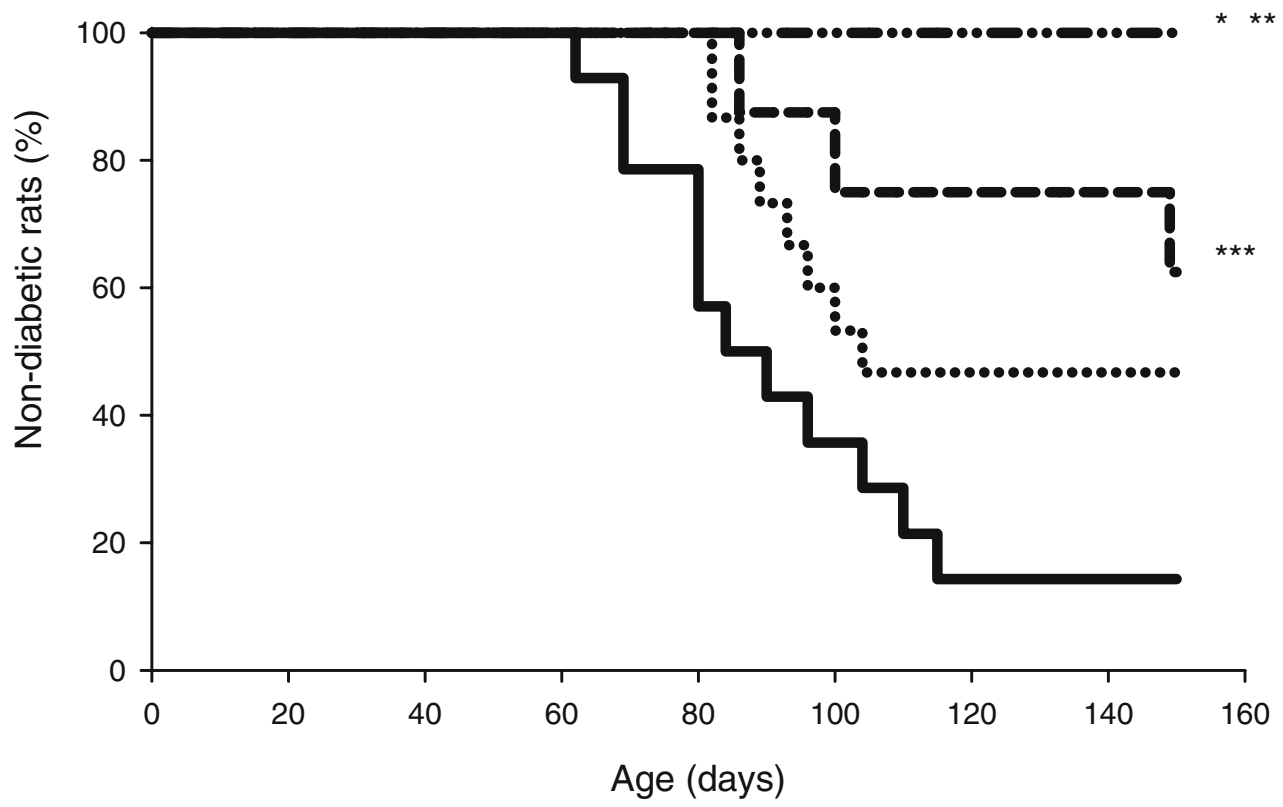


95, 92 and 93\% was seen for Bac303, Erec482, Mib661 and Lab158, respectively (data not shown).

\section{Antibiotic treatment and diabetes development}

Figure 2 shows that control rats receiving only CON developed diabetes from 60 days of age and eventually $86 \%$ of the rats developed diabetes (12 out of 14 rats). However, if rats received antibiotics with the CON diet the diabetes incidence decreased to $37.5 \%$ (three out of eight rats) $(p=0.02)$. Interestingly, antibiotic treatment also delayed diabetes onset by 30 days $(p=0.004)$. In the $\mathrm{HC}$ diet group, $53 \%$ of the animals developed diabetes ( 8 out of 15 rats) $(p=0.08)$. However, diabetes onset was significantly delayed by 25 days $(p=0.04)$. Strikingly, combining antibiotic treatment and $\mathrm{HC}$ diet totally protected against diabetes in the BB-DP rat; none of the ten rats developed diabetes ( $p=0.01$ compared with the diet control group; $p=0.002$ compared with the group receiving CON alone).

Insulitis

All diabetic animals displayed severe insulitis (data not shown), consistent with the finding that clinical diabetes becomes manifest when $80 \%$ of the islets are destroyed. The non-diabetic rats in all groups did not display insulitis (score 1 ) or only very mild insulitis (score 2) (data not shown).

\section{Discussion}

Our data strongly support the notion that the composition of the gut flora is indeed involved in the development of type 1 diabetes. The finding that the faecal flora differs before the onset of the disease between rats that will and those that will not develop diabetes suggests that the flora might have a causative rather than a secondary role in the pathology of diabetes.

Antibiotic modulation of the intestinal flora in our BBDP rat model for type 1 diabetes delayed and lowered the diabetes incidence significantly. A combination of antibiotic treatment and $\mathrm{HC}$ diet was able to totally prevent diabetes in the BB-DP rat. Modulation of the intestinal flora by antibiotic treatment could lower the antigenic load and therefore reduce diabetes incidence. However, antibiotic treatment (fusidic acid or cyclosporin A) has also been shown to prevent the lymphocyte-stimulatory activities of the cytokines IL-1 and IL-6 [10]. Antibiotic treatment therefore could also have a direct effect on the pancreas. Furthermore, protective diets have been shown to alter the bacterial composition [3]. Similarly, we find differences in the intestinal flora before the onset of diabetes in unmanipulated BB-DP rats between rats that will or will not develop diabetes eventually.

Modulating the composition of the intestinal flora by antibiotic treatment and/or HC diet selectively removes some commensals while others are able to grow, thereby not only reducing the overall antigenic load but reducing the antigenic load of a given specificity as well.

We propose that the (specific) antigenic load in the intestine is important for the outcome of diabetes and that this antigenic load has a different effect on the immune system in different periods in life. Analysis of the complex interactions between antigenic load, the composition of the intestinal flora and the (gut) immune system in type 1 diabetes could well lead to new insights into this disease. Furthermore, this knowledge could possibly provide an important basis for the development of future preventive measures.

Acknowledgement This work was supported by a grant from the Dutch Diabetes Research Foundation.

\section{References}

1. Scott FW (1996) Food-induced type 1 diabetes in the BB rat. Diabetes Metab Rev 12:341-359

2. Scott FW, Cloutier HE, Kleemann R et al (1997) Potential mechanisms by which certain foods promote or inhibit the development of spontaneous diabetes in BB rats: dose, timing, early effect on islet area, and switch in infiltrate from Th1 to Th2 cells. Diabetes 46:589-598

3. Hansen AK, Ling F, Kaas A, Funda DP, Farlov H, Buschard K (2005) Diabetes preventive gluten-free diet decreases the number of caecal bacteria in non-obese diabetic mice. Diabetes Metab Res Rev 22:220-225

4. Funda DP, Kaas A, Bock T, Tlaskalova-Hogenova H, Buschard K (1999) Gluten-free diet prevents diabetes in NOD mice. Diabetes Metab Res Rev 15:323-327

5. Brugman S, Klatter FA, Visser J, Bos NA, Elias D, Rozing J (2004) Neonatal oral administration of DiaPep277, combined with hydrolysed casein diet, protects against Type 1 diabetes in BB-DP rats. An experimental study. Diabetologia 47:1331-1333

6. Kolb H, Worz-Pagenstert U, Kleemann R, Rothe H, Rowsell P, Scott FW (1996) Cytokine gene expression in the BB rat pancreas: natural course and impact of bacterial vaccines. Diabetologia 39:1448-1454

7. Buschard K, Pedersen C, Hansen SV, Hageman I, Aaen K, Bendtzen K (1992) Anti-diabetogenic effect of fusidic acid in diabetes prone BB rats. Autoimmunity 14:101-104

8. van der Waaij LA, Harmsen HJ, Madjipour M et al (2005) Bacterial population analysis of human colon and terminal ileum biopsies with $16 \mathrm{~S}$ rRNA-based fluorescent probes: commensal bacteria live in suspension and have no direct contact with epithelial cells. Inflamm Bowel Dis 11:865-871

9. Salzman NH, de Jong H, Paterson Y, Harmsen HJ, Welling GW, Bos NA (2002) Analysis of 16S libraries of mouse gastrointestinal microflora reveals a large new group of mouse intestinal bacteria. Microbiology 148:3651-3660

10. Bendtzen K, Diamant M, Horn T, Pedersen C, Buschard K (1992) Effect of fusidic acid on interleukin-1 (IL-1)- and IL-6-induced pancreatic beta-cell functions in rats. J Endocrinol 132:345-352 